\title{
HIGHLIGHTS
}

PEDIATRIC ONCOLOGY

\section{Pulsed treatment in continuation therapy improves disease-free survival in children with hematologic malignancies}

Continuation therapy, after the induction stage, has long been an established method for the treatment of childhood acute lymphoblastic leukemia (ALL). Historically, it had been shown that patients treated with additional therapies during this continuation period had an improved disease-free survival. However, by the late 1990s, most average-risk patients were being treated with the newer Berlin-Frankfurt-Münster (BFM) regimen and the question was raised as to whether the old adage remained true that more treatment in continuation was better.

\section{4 ...the EORTC-CLC group have reintroduced pulsed treatment as the standard continuation therapy... 77}

As the first author of the present study, Barbara De Moerloose described, "the international BFM study group initiated a cooperative study ... to evaluate the potential benefit of vincristine-

dexamethasone pulses during continuation therapy." Although the Children's Leukemia Group of the EORTC participated in the BFM trial, they additionally randomized for other factors and included a long follow up.

Interestingly, the efficacy end points had different results. In the BFM trial (follow up for 4.8 years), no benefit was observed from the inclusion of pulsed therapy in continuation treatment and the randomization was halted because of futility. However, in the EORTC study, after 6.3 years of follow up, a very different conclusion has been drawn.

In the 411 average-risk ALL and nonHodgkin lymphoma patients, those randomized to receive the pulses of vincristine and corticosteroids had a significantly improved 6-year disease-free survival rate compared with those not receiving the pulses ( $90.6 \%$ versus $82.8 \%$; $P=0.027)$. The difference observed in overall survival was not significant. The effect of the pulses on disease-free survival was more pronounced in girls than in boys (hazard ratio 0.24 versus 0.71 ).

More work needs to be done to define the best therapy. De Moerloose explains, "future research will have to elucidate which corticosteroid in the pulses will yield the best result and what should be the optimal number of pulses to be given."

However, the impact of these results can be easily highlighted by the fact that the EORTC-CLC group have reintroduced pulsed treatment as the standard continuation therapy stage of average-risk patients undergoing EORTC studies.

Rebecca Kirk

Original article De Moerloose, B. et al. Improved outcome with pulses of vincristine and corticosteroids in continuation therapy of children with average risk acute lymphoblastic leukemia (ALL) and lymphoblastic non-Hodgkin lymphoma (NHL): report of the EORTC randomized phase 3 trial 58951. Blood 116, 36-44 (2010) 